\title{
INFLUENCE OF LOCAL POTENTIAL ON EXCITON SPLITTING IN HIGHLY DILUTED $\mathrm{Cd}_{1-x} \mathrm{Mn}_{x} \mathrm{Te}$
}

\author{
Ł. Klopotowski, M. Herbich, W. Mac, A. Stachow-Wójcik \\ AND A. TWARDOWSKI
}

Institute of Experimental Physics, Warsaw University, IIoża 69, 00-681 Warsaw, Poland

\begin{abstract}
We investigated magnetoreflectance and magnetization of highly diluted bulk $\mathrm{Cd}_{1-x} \mathrm{Mn}_{x}$ Te crystals $0.2 \% \leq x \leq 10 \%$. The exchange constant in terms of mean field approximation and virtual crystal approximation (the ratio of the heavy hole exciton splitting to mean spin per unit cell) was evaluated and found $x$-dependent. This deviation from the mean field approximation and virtual crystal approximation prediction is caused by the local potential introduced by Mn ions. We discuss the problem within a Wigner-Seitz approach and within a model of magnetic and chemical disorder based on the alloy theory.
\end{abstract}

PACS numbers: 71.70.Gm, 75.10.Dg, 75.50.Pp

The mean field approximation (MFA) together with virtual crystal approximation (VCA) were successfully used to describe $s, p-d$ exchange interactions in diluted magnetic semiconductors (DMS). However, experiments done on highly diluted $\mathrm{Cd}_{1-x} \mathrm{Mn}_{x} \mathrm{~S}$ and later on $\mathrm{Zn}_{1-x} \mathrm{Mn}_{x}$ Se showed that the above approach is insufficient $[1,2]$. In both experiments the valence band exchange integral evaluated in terms of MFA and VCA increases with decreasing $x$. In the framework of MFA and VCA the valence band exchange constant $N_{0} \beta$ describes only the magnitude of exchange interaction and does not depend on $x$. A magnetic impurity introduces to the crystal a local potential consisting of two terms: spin independent chemical shift and spin dependent exchange potential. For appreciable Mn content (roughly larger than a few \%) delocalized band carrier interacts with a large number of $\mathrm{Mn}$ ions and the volume interaction averaging over the crystal volume done in terms of VCA is appropriate. But in a highly dilute limit a carrier may interact with only a few ions and in the case of an attractive impurity potential can be almost bound. This results in larger than VCA-predicted exciton splitting. As a consequence, the evaluated exchange constant (in terms of VCA) is found $x$-dependent. Its absolute value increases with decreasing $x$. On the other hand, for a repulsive impurity potential slightly lower than the ones predicted by VCA exciton splittings are expected. 
The first theoretical model describing this effect (using $x$-independent $N_{0} \beta$ ) was based on a cellular Wigner-Seitz approach, which forced an assumption of periodic distribution of $\mathrm{Mn}$ impurities [3]. Recently the model of chemical and magnetic disorder has been developed [4]. The calculations were performed in the saturation limit, i.e., assuming that all impurities' spins are aligned. Both approaches show that the departure from VCA depends on the magnitude of the local potential and is only significant for heavy carriers $[3,4]$. Hence the VCA will remain valid for the conduction band and only the exchange constant for the valence band will reveal the $x$-dependence. The calculations show also that the effect of an attractive potential is supposed to be much stronger than for a repulsive one.

Both the periodic superlattice and the disorder models have been successfully used to interpret the experimental data for $\mathrm{Cd}_{1-x} \mathrm{Mn}_{x} \mathrm{~S}[3,4]$ and $\mathrm{Zn}_{1-x} \mathrm{Mn}_{x} \mathrm{Se}$ [2]. In both of them the considered effect resulted from the strong, attractive spin independent component. Experiments on a material with a prominent repulsive potential could serve as a crucial test of the proposed model. Estimations showed that the widely studied $\mathrm{Cd}_{1-x} \mathrm{Mn}_{x} \mathrm{Te}$ is such a material.

Therefore we measured magnetoreflectance in the free exciton range of bulk $\mathrm{Cd}_{1-x} \mathrm{Mn}_{x} \mathrm{Te}$ crystals with a manganese molar fraction $0.2 \% \leq x \leq 10 \%$, in a magnetic field up to $5 \mathrm{~T}$ and at $T=2.0 \mathrm{~K}$. Due to the high quality of the crystals all four excitonic lines were resolved for $x$ as low as $0.4 \%$ and their splitting in the magnetic field was determined (see Fig. 1).

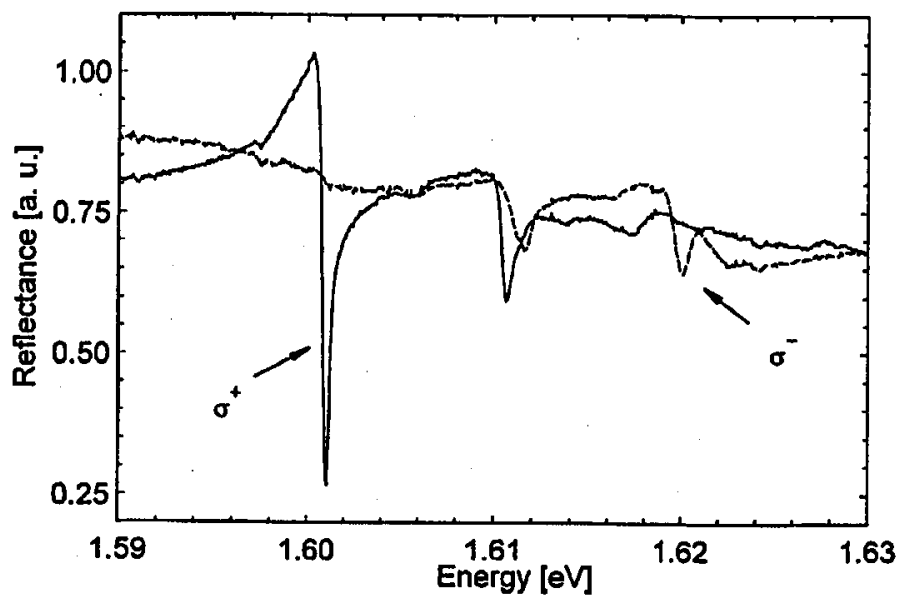

Fig. 1. Magnetoreflectance spectrum of $\mathrm{Cd}_{0.99} \mathrm{Mn}_{0.01} \mathrm{Te}$ for $B=5 \mathrm{~T}$.

Under the same temperature and magnetic field conditions magnetization was measured using a SQUID magnetometer. The modified Brillouin function with two parameters $S_{0}$ and $T_{0}$

$$
\mathcal{M}=\frac{N_{\mathrm{A}}}{m} g \mu_{\mathrm{B}} x S_{0} B_{S}\left[\frac{g S \mu_{\mathrm{B}} B}{k_{\mathrm{B}}\left(T+T_{0}\right)}\right]
$$


(where $m$ is the mass of $\mathrm{Cd}_{1-x} \mathrm{Mn}_{x} \mathrm{Te}$ "molecule") was fitted to the obtained data. Good agreement between this model and the experiment was observed.

Mn concentration for samples of $x \geq 1 \%$ was determined from the energy gap concentration dependence $E_{\mathrm{g}}(x)$ [5]. Below $1 \% x$ was obtained from the measured magnetization. (In Eq. (1) we put $S_{0}=\frac{5}{2}$ and $T_{0}=0$ as in this case the ions can be considered as noninteracting [5].)

Mean Mn spin per unit cell was calculated using the relation

$$
x\left\langle-S_{z}\right\rangle=\mathcal{M} \frac{m}{g \mu_{\mathrm{B}}}
$$

with $g=2$.

Following MFA and VCA we evaluated the difference between exchange constants for the conduction and valence band as the ratio of the heavy hole exciton splitting to the mean spin per unit cell

$$
N_{0} \alpha-N_{0} \beta=\frac{\Delta E_{\mathrm{hh}}}{x\left\langle-S_{z}\right\rangle} \text {. }
$$

We evaluated $N_{0} \alpha$ from the equations giving band splitting and we found it $x$-independent (within experimental accuracy). The calculated mean value gave $N_{0} \alpha=0.20 \pm 0.02 \mathrm{eV}$. We decided to use the value of $N_{0} \alpha=0.22 \mathrm{eV}$ obtained in experiments on $\mathrm{Cd}_{1-x} \mathrm{Mn}_{x} \mathrm{Te}$ with a higher $x$ [6] arguing that the value should be free of any local potential effect.

Next, $N_{0} \beta^{\mathrm{VCA}}$ was calculated. The dependence on $x$ was found to be opposite to that of $\mathrm{Cd}_{1-x} \mathrm{Mn}_{x} \mathrm{~S}$ and $\mathrm{Zn}_{1-x} \mathrm{Mn}_{x} \mathrm{Se}$, the absolute value of the exchange constant was decreasing with decreasing $x$. The effect was found to be much smaller (see Fig. 2) than in $\mathrm{Zn}_{1-x} \mathrm{Mn}_{x} \mathrm{Se}$, where $N_{0} \beta^{\mathrm{VCA}}$ varied by a factor of 2 .

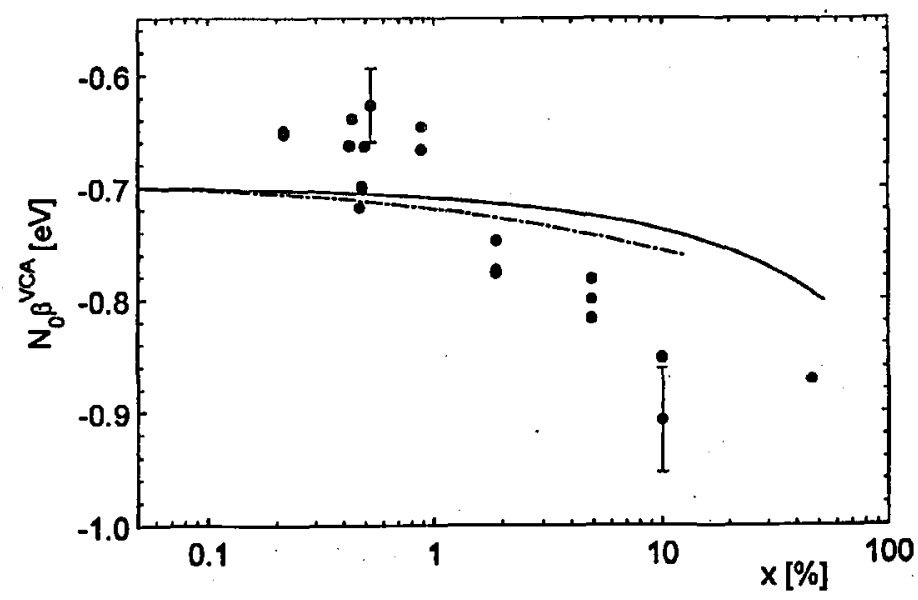

Fig. 2. Valence band exchange constant evaluated in terms of virtual crystal approximation with the model calculations. The solid line represents the model of magnetic and chemical disorder, the dashed one - the model of periodic superlattice of impurities (see the text). 
We performed calculations within both approaches described above. Only qualitative agreement with the experimental data was obtained. Still both models predict even weaker $x$-dependence of VCA-evaluated $N_{0} \beta$ than the one observed. Calculations done within the periodic superlattice model yielded $N_{0} \beta=-0.77 \mathrm{eV}$ and calculations in terms of the disorder model gave $N_{0} \beta=-0.88 \mathrm{eV}$.

\section{References}

[1] V.G. Abrammishvili, S.I. Gubarev, A.V. Komarov, S.M. Ryabchenko, Fiz. Tverd. Tela 26, 1095 (1984) [Sov. Phys. Solid State 26, 666 (1984)]; S.I. Gubarev, M.G. Tyazlov, Pis'ma Zh. Eksp. Teor.Fiz. 44, 385 (1986) [JETP Lett. 44, 494 (1986)]; Pis'ma Zh. Eksp. Teor. Fiz. 48, 437 (1988) [JETP Lett. 48, 481 (1988)]; Fiz. Tverd. Tela 32, 635 (1990) [Sov. Phys. Solid State 32, 373 (1990)]; M. Nawrocki, J.P. Lascaray, D. Coquillat, M. Demianiuk, in: Materials for Infrared Detectors and Sources, Eds. R.L. Aggarwal et al., MRS Symposia Proc. No. 89, Materials Research Society, Pittsburgh 1987, p. 65.

[2] M. Herbich, W. Mac, A. Stachow, A. Twardowski, J. Tworzydło, M. Demianiuk, Acta Phys. Pol. A 90, 813 (1996).

[3] C. Benoit à la Guillame, D. Scalbert, T. Dietl, Phys. Rev. B 46, 9853 (1992).

[4] J. Tworzydło, Phys. Rev. B 50, 14591 (1994); Solid State Commun. 94, 821 (1995).

[5] J.K. Furdyna, J. Appl. Phys. 64, R29 (1988).

[6] J.A. Gaj, R. Planel, G. Fishman, Solid State Commun. 29, 435 (1979). 